\title{
Liver mitochondrial membrane crosslinking and destruction in a rat model of Wilson disease
}

\author{
Hans Zischka, ${ }^{1}$ Josef Lichtmannegger, ${ }^{1}$ Sabine Schmitt, ${ }^{1}$ Nora Jägemann, ${ }^{1}$ Sabine Schulz, ${ }^{1}$ \\ Daniela Wartini, ${ }^{1}$ Luise Jennen, ${ }^{2}$ Christian Rust, ${ }^{3}$ Nathanael Larochette, ${ }^{4}$ Lorenzo Galluzzi, ${ }^{4,5,6}$ \\ Veronique Chajes, ${ }^{5,7}$ Nathan Bandow, ${ }^{8}$ Valérie S. Gilles, ${ }^{8}$ Alan A. DiSpirito, ${ }^{8}$ Irene Esposito, ${ }^{2,9}$ \\ Martin Goettlicher, ${ }^{1,10}$ Karl H. Summer, ${ }^{1}$ and Guido Kroemer ${ }^{4,11,12,13,14}$
}

\begin{abstract}
${ }^{1}$ Institute of Toxicology and 2Institute of Pathology, Helmholtz Center Munich, German Research Center for Environmental Health, Neuherberg, Germany. ${ }^{3}$ Department of Medicine 2 - Grosshadern, University of Munich, Munich, Germany. ${ }^{4}$ INSERM, U848, 5 Institut Gustave Roussy, and ${ }^{6}$ Université Paris-Sud XI, Villejuif, France. ${ }^{7}$ International Agency for Research on Cancer, Lyon, France. ${ }^{8}$ Department of Biochemistry, Biophysics and Molecular Biology, lowa State University, Ames, Iowa, USA. Institute of Pathology and ${ }^{10}$ Institute of Toxicology and Environmental Hygiene, Technical University Munich, Munich, Germany. ${ }^{11}$ Metabolomics Platform, Institut Gustave Roussy, Villejuif, France. ${ }^{12}$ Centre de Recherche des Cordeliers, Paris, France. ${ }^{13}$ Pôle de Biologie, Hôpital Européen Georges Pompidou, AP-HP, Paris, France. ${ }^{14}$ Université Paris Descartes, Paris 5, Paris, France.
\end{abstract}

\begin{abstract}
Wilson disease (WD) is a rare hereditary condition that is caused by a genetic defect in the copper-transporting ATPase ATP7B that results in hepatic copper accumulation and lethal liver failure. The present study focuses on the structural mitochondrial alterations that precede clinical symptoms in the livers of rats lacking Atp7b, an animal model for WD. Liver mitochondria from these $A t p 7 b^{-/-}$rats contained enlarged cristae and widened intermembrane spaces, which coincided with a massive mitochondrial accumulation of copper. These changes, however, preceded detectable deficits in oxidative phosphorylation and biochemical signs of oxidative damage, suggesting that the ultrastructural modifications were not the result of oxidative stress imposed by copper-dependent Fenton chemistry. In a cell-free system containing a reducing dithiol agent, isolated mitochondria exposed to copper underwent modifications that were closely related to those observed in vivo. In this cell-free system, copper induced thiol modifications of three abundant mitochondrial membrane proteins, and this correlated with reversible intramitochondrial membrane crosslinking, which was also observed in liver mitochondria from $A t p 7 b^{-/-}$rats. In vivo, copper-chelating agents reversed mitochondrial accumulation of copper, as well as signs of intra-mitochondrial membrane crosslinking, thereby preserving the functional and structural integrity of mitochondria. Together, these findings suggest that the mitochondrion constitutes a pivotal target of copper in WD.
\end{abstract}

\section{Introduction}

Wilson disease (WD) is an autosomal recessive inherited fatal disorder (1). Loss-of-function mutations in the ATP7B gene, coding for a copper-transporting ATPase, lead to massive copper accumulation, primarily in the liver, typically exceeding a hepatic copper content of $250 \mu \mathrm{g} / \mathrm{g}$ dry weight (2-4). Prominent clinical features of WD include chronic hepatitis, progressive liver cirrhosis, and hepatic failure (4). Copper accumulation can be prevented by oral administration of copper-chelating agents such as D-penicillamine (D-PA) (4-6). In conditions of fulminant hepatic failure, liver transplantation constitutes the only therapeutic option (4).

Although WD and the causative hepatic copper overload were described as early as in 1912 and 1948 , respectively $(7,8)$, the mechanisms by which excess of copper damages hepatocytes are poorly understood $(9,10)$. It has been repeatedly theorized that copper-mediated oxidative damage would account for the death of hepatocytes $(4,11,12)$, based on the intrinsic ability of copper to undergo redox cycling between $\mathrm{Cu}(\mathrm{II})$ and $\mathrm{Cu}(\mathrm{I})(13,14)$. Reactions between free copper and oxygen species can produce hydroxyl radicals via Fenton reactions, resulting in damage of cellular lipids,

Conflict of interest: The authors have declared that no conflict of interest exists. Citation for this article: J Clin Invest. 2011;121(4):1508-1518. doi:10.1172/JCI45401 proteins, and nucleic acids (13-15). In fact, several studies on WD patients or appropriate animal models have reported oxidative damage of these biomolecules $(14,15)$. However, other studies have noted the absence of free intracellular copper, which is inconsistent with the free radical theory of WD pathogenesis $(16,17)$. These studies have demonstrated that copper is bound to either proteins or low-molecular-weight ligands and hence unavailable for uncontrolled large-scale "oxidative fires" (16-19), launching the quest for alternative mechanisms of pathogenesis.

Mitochondria play a prominent role in lethal signaling (20-22). Sternlieb et al. reported prominent structural alterations of mitochondria in liver tissues of WD patients (23-25). These structural alterations are recapitulated by a rat model that bears an Atp $7 b$ deletion, the Long-Evans Cinnamon (LEC) rat $(26,27)$. Importantly, the structural alterations of mitochondria occur at an early stage, when clinical signs of hepatic failure are still absent $(23,24)$.

The mechanistic link between copper accumulation and mitochondrial structural and functional alterations has been a conundrum. Sternlieb, for instance, failed to observe any correlation between the type/extent of mitochondrial abnormality and the hepatic copper concentration, be it in WD patients or in $A t p 7 b^{-1}$ LEC rats $(24,28)$. Sokol and coworkers, however, reported that excessive copper feeding does cause structural and functional deficits in rat liver mitochondria $(9,29)$. 

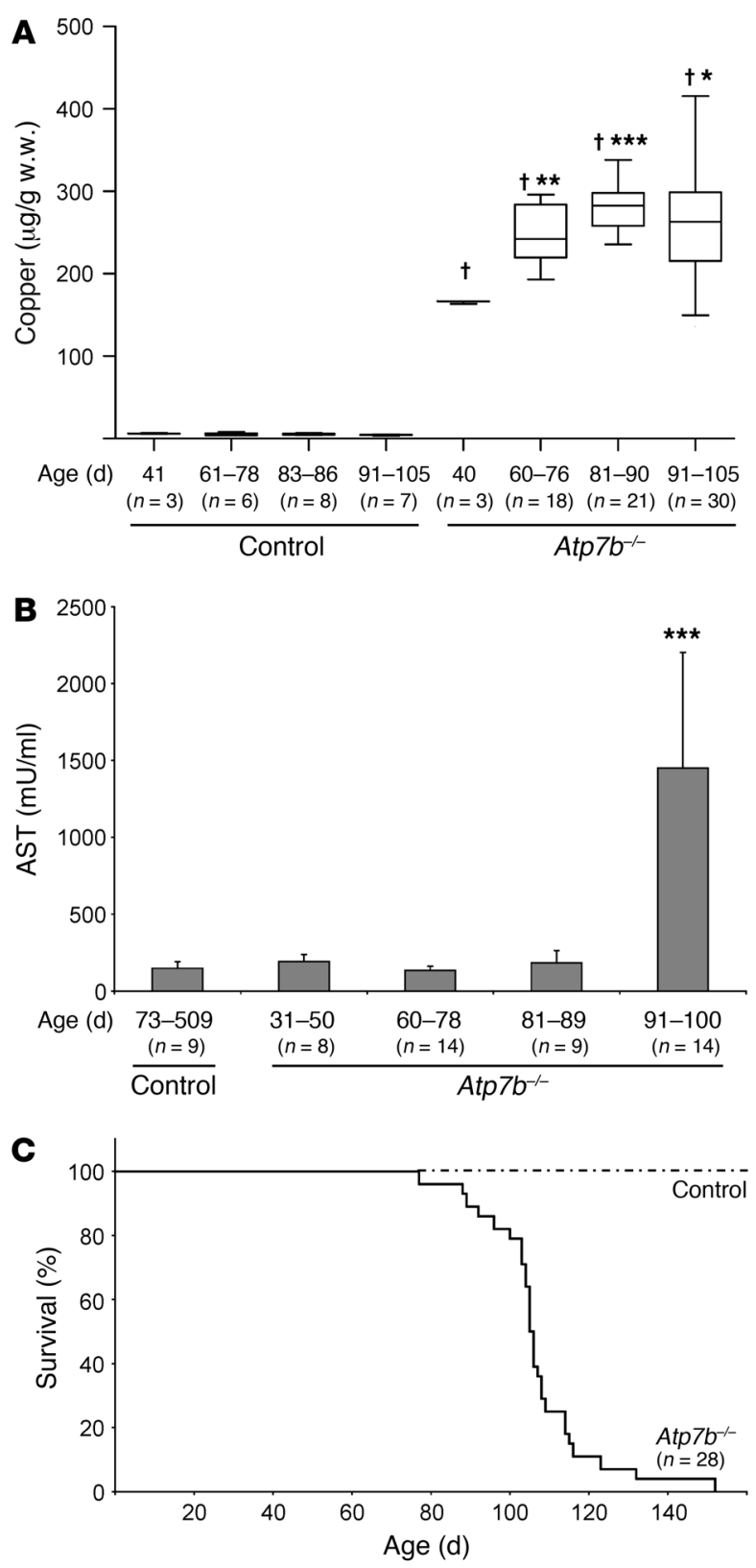

How might copper accumulation lead to mitochondrial dysfunction? Oxidative membrane damage, which has been suggested to cause these mitochondrial alterations $(15,29,30)$, typically entails mitochondrial permeability transition (MPT), characterized by a concomitant swelling of the organelles that ruptures their outer membranes $(31,32)$. However, WD mitochondria exhibit a "contraction-like" phenotype, especially in the early disease phase, with dense matrices and enlarged intermembrane spaces but intact outer membranes (24), casting an MPT-mediated pathogenesis into doubt.

Driven by these premises and unknowns, we reexamined the kinetics of ultrastructural alterations affecting hepatocyte mitochondria from $A t p 7 b^{-/-}$rats. Here, we report that mitochondrial ultrastructure progressively changes as copper accumulates within this organelle in vivo or in vitro, when mitochondria are exposed to copper, finally culminating in mitochondrial destruction. Moreover, we examined the biochemical mechanisms through

\section{Figure 1}

Liver copper accumulation in $A t p 7 b^{-/-}$rats is accompanied by organ failure and animal death. (A) In Atp $7 b^{-/-}$animals, hepatic copper progressively accumulates, reaching a plateau around 70 days of age, in contrast to control rats (i.e., Atp $7 b^{+/-}$and $A t p 7 b^{+/+}$rats). Boxes display the middle $50 \%$ of the data, restricted by the 25 th and 75 th percentiles. The line within the box represents the median. Maximum and minimum values are represented by the whiskers. ${ }^{\dagger} P<0.001$ versus respective control age group; ${ }^{*} P<0.05$, ${ }^{\star \star} P<0.01$, ${ }^{* \star *} P<0.001$ versus the youngest $A t p 7 b^{-/-}$age group. Liver copper was normalized to liver wet weight (w.w.). (B) Liver damage, as assessed by an increase in AST activity in serum, occurs abruptly in $A t p 7 b^{-/}$rats around 90 days of age ( $n=$ numbers of animals in the different age groups). ${ }^{* *} P<0.001$. (C) Untreated $A t p 7 b^{-1-}$ rats (strain LPP) die between 90 and 120 days of age, with a median survival of 106 days.

which copper induces these sequential alterations and found that copper can induce crosslinking of mitochondrial proteins within and between distinct mitochondrial membranes, thus explaining an important step of the pathogenesis of WD.

\section{Results}

A defect in the Wilson gene causes abrupt onset of hepatic failure in rats. LEC rats and the derived LPP rat strain share the same autosomal recessive genetic defect in the copper-transporting ATPase Atp7b (33). Consequently, in homozygous Atp $7 b^{-/-}$animals, hepatic copper progressively accumulated, reaching a plateau at around 70 days of age (Figure 1A). Heterozygous $A t p 7 b^{+/-}$and wild-type $A t p 7 b^{+/+}$rats did not accumulate hepatic copper and hence served as control animals in this study (Figure 1A). In sharp contrast to the initially increasing hepatic copper load, $A t p 7 b^{-/-}$animals developed laboratory signs of liver damage rather late and abruptly, starting at around 90 days of age. These signs of hepatotoxicity included increased plasma levels of aspartate aminotransferase (AST) (average values per group > $200 \mathrm{mU} / \mathrm{ml}$; Figure 1B) and bilirubin (>0.5 mg/dl; Supplemental Figure 1; supplemental material available online with this article; doi:10.1172/JCI45401DS1). Diseased $A t p 7 b^{-/}$animals, which we termed "clinically apparent," died within 30 days after detection of elevated plasma AST concentrations (Figure 1C) if left untreated.

Copper-induced mitochondrial ultrastructural changes occur before manifestation of liver failure and are related to copper accumulation. Mitochondria from Atp $7 b^{-/-}$hepatocytes displayed rounded cristae and enlarged intermembrane spaces with membranous relics (Figure 2, B-D, and Supplemental Figure 2), as previously described in WD patients and $A t p 7 b^{-/-}$rats $(23-25,28)$. We classified these mitochondrial alterations into 3 categories: normal (Figure 2A), altered (mitochondria with detectable enlargements in cristae and/or intermembrane spaces; Figure 2, B and C), and severely condensed (mitochondria with massive matrix condensation, enlargement of cristae and intermembrane spaces, and shape deterioration; Figure 2D). Electron micrographs from mitochondria that were isolated from $A t p 7 b^{-1-}$ animals and purified by density gradient centrifugation and free-flow electrophoresis (34) revealed an age-dependent quantitative increase in mitochondrial damage (Figure 2, B-E, and Supplemental Figure 2, C-E). This damage was apparent at 50 days of age and continually increased up to the age of 90 days (Figure 2E). Moreover, a continually increasing copper load paralleled these structural alterations in $A t p 7 b^{-/-}$mitochondria (Figure $2 \mathrm{~F})$. The intramitochondrial copper concentration was twice as 

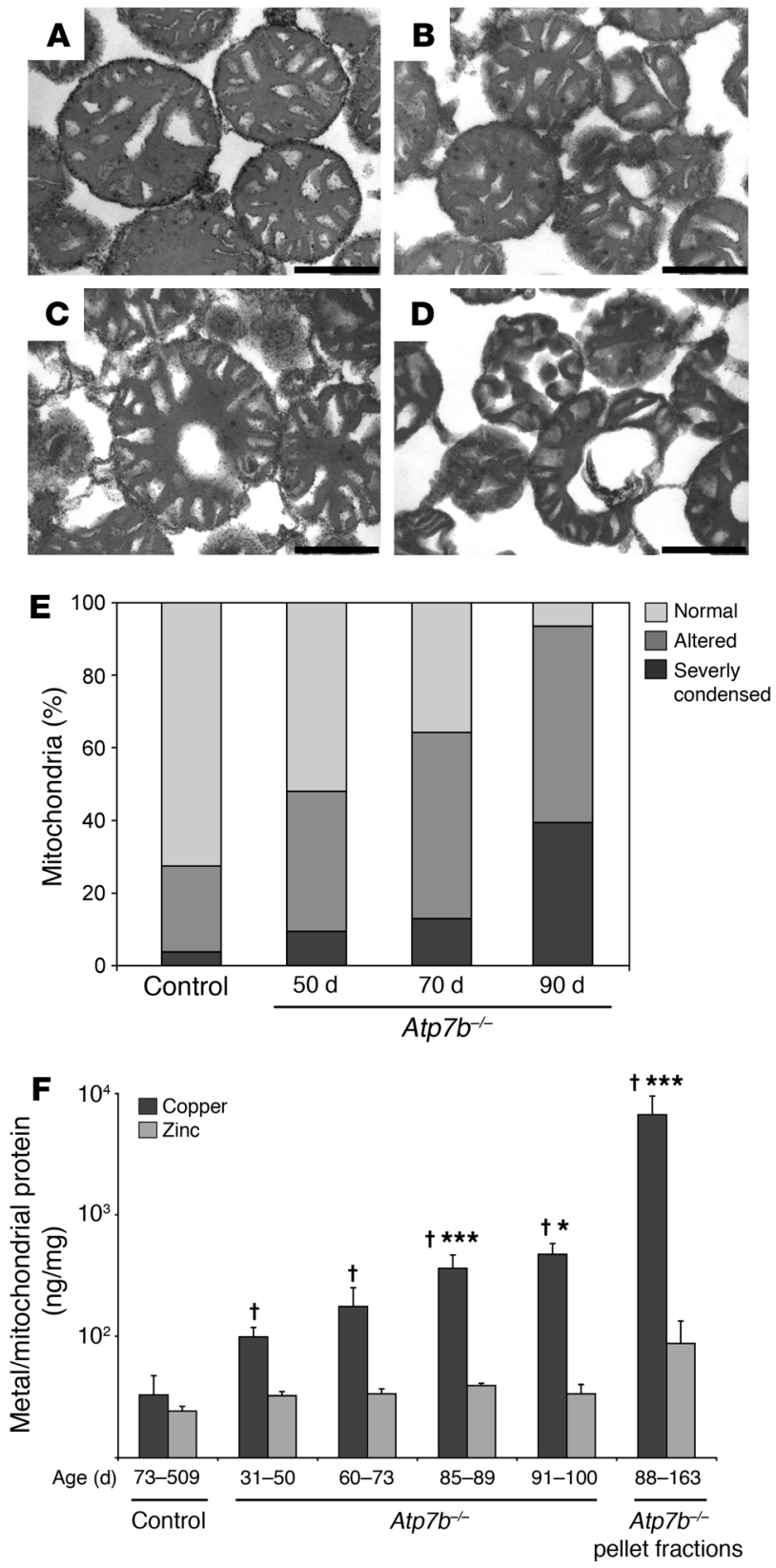

high as that of controls at 30-50 days of age and reached 10 times the control level at the age of 90 days (Figure 2F).

Isopycnic purification of mitochondria isolated from control or young $A t p 7 b^{-1-}$ rats reproducibly yielded a single major mitochondrial population (34). Importantly, an additional fraction of mitochondria appeared in the pellet of density-centrifuged liver mitochondria from clinically apparent $A t p 7 b^{-/-}$but not control rats (Figure 3, A-C, Supplemental Figure 3, and see below). This pellet fraction contained mitochondria with greatly enlarged intermembrane spaces (Figure 3A) and grossly transformed mitochondria with matrix remnants gathering at the membrane (Figure $3 \mathrm{~B}$ and Supplemental Figure 3A) frequently associated with massive electron-dense deposits (Figure 3C). Electron micrographs of intermediates of these transformed structures validated their mitochondrial origin, and immunochemical analyses confirmed the

\section{Figure 2}

Structural alterations coincide with increasing copper load in Atp $7 b^{-/-}$ mitochondria. Electron micrographs of liver mitochondria isolated from (A) control rat, displaying normal mitochondria; (B) Atp $7 b^{-/-}$rat (age 50 days) with altered morphology characterized by enlarged cristae and slightly altered organelle shapes; (C) Atp $7 b^{-/-}$rat (age 70 days); (D) Atp $7 b^{-/-}$rat (age 90 days) with severely condensed mitochondria characterized by greatly enlarged intermembrane spaces and shape alterations. Scale bars: $0.5 \mu \mathrm{m}$. (E) Structural alterations in isolated $A t p 7 b^{-/-}$mitochondria increase with age relative to control mitochondria. Quantification at each time point was based on electron micrographs showing around 200 mitochondria. (F) Copper steadily increases in isolated $A t p 7 b^{-/-}$mitochondria, whereas zinc levels remain relatively constant. Note the logarithmic scale, demonstrating an approximately 10 -fold increase in copper values in 91- to 100-day-old Atp $7 \mathrm{~b}^{-/-}$mitochondria and approximately 200 -fold increase in the pellet fraction in comparison to control mitochondria. Mitochondria were isolated from control animals at age 73-509 days

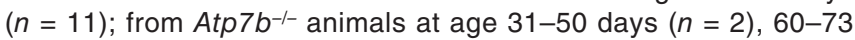
days ( $n=13), 85-89$ days $(n=6), 91-100$ days $(n=17)$; and from the pellet fractions of clinically apparent $A t p 7 \mathrm{~b}^{-/-}$animals at age 88-163 days $(n=11)$. ${ }^{*} P<0.05,{ }^{* *} P<0.001$ versus the preceding age group; ${ }^{\dagger} P<0.001$ versus control; $t$ test.

mitochondrial presence in this fraction (Supplemental Figure 3). The copper content of this fraction exceeded control levels by more than 200-fold (note the logarithmic scale in Figure 2F).

Oxidative damage in mitochondria occurs at a late stage. Based on previous studies implicating copper-mediated oxidative stress as the primary etiological determinants of $\operatorname{WD}(4,14,35)$, we assessed the oxidative damage of mitochondria isolated from $A t p 7 b^{-1-}$ rats by measuring the ROS-driven decline in mitochondrial aconitase activity (Figure 4A) and (poly)unsaturated mitochondrial fatty acids (Figure 4B). No signs of oxidative damage were detectable before the disease became clinically apparent (Figure 4, A and B). Accordingly, selected mitochondrial electron-transporting respiratory complexes from $A t p 7 b^{-/-}$animals were as enzymatically active as those from control rat livers (Figure 4C). In contrast, band and pellet mitochondrial fractions isolated from clinically apparent $A t p 7 b^{-1-}$ animals displayed slightly altered and severely impaired enzymatic activities, respectively (Figure 4C).

Simulation of pathological mitochondrial alterations in a cell-free system. In an attempt to reproduce the ultrastructural perturbations that characterize $A t p 7 b^{-/-}$mitochondria, we employed a cell-free system in which isolated control mitochondria were exposed to copper. At nonreducing conditions, copper induced a decrease in light scattering at $540 \mathrm{~nm}$, which is referred to as "mitochondrial swelling." This swelling was similar to that induced by calcium, the classical MPT inducer (Figure 5A), but could not be inhibited by cyclosporine A (CsA), the prototypic MPT inhibitor, in conditions in which it readily suppressed calcium-induced swelling (Supplemental Figure 4A). Since copper ions are not free but largely bound to the thiol groups of proteins in vivo (36), we measured the putative MPT-inducing effects of copper in the presence of thiol-bearing antioxidants. The mono-thiol glutathione (GSH), as well as the two bivalent thiols DTT and dithioerythritol (DTE), completely blocked copper-induced swelling (Figure 5A).

In the presence of DTT, repetitive copper dosing instantaneously induced a staircase-like increase in absorbance, indicative of mitochondrial contraction (Figure 5B and Supplemental Figure 4B). This phenomenon could be fully reversed by repetitively adding 

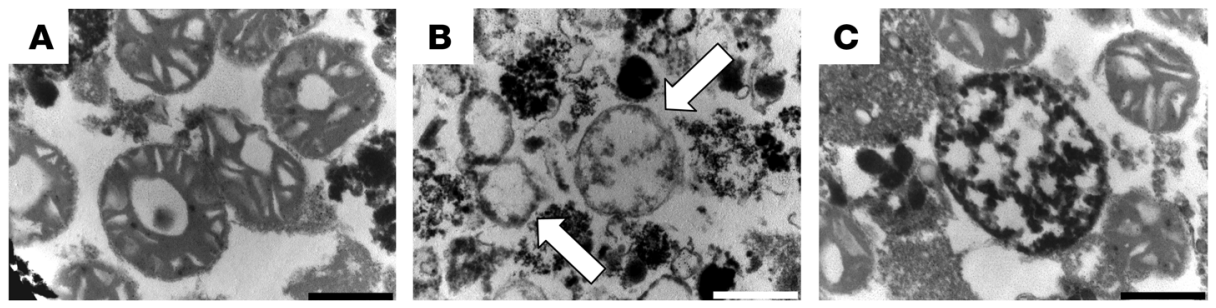

Figure 3

Clinically apparent $A t p 7 b^{-/-}$livers contain severely damaged mitochondria. Electron micrographs of pellet fractions isolated from $A t p 7 b^{-/-}$livers as WD becomes clinically apparent. This pellet fraction contained mitochondria (A) with greatly enlarged intermembrane spaces and $(\mathbf{B})$ mitochondria with matrix remnants gathering at the membrane (arrows), which were frequently associated with massive electron-dense deposits (C). Scale bars: $0.5 \mu \mathrm{m}$.

equimolar doses of the copper chelator bathocuproine disulfonic acid (BCDS) (Figure 5B) or by the peptide methanobactin (Supplemental Figure 4B), which has a high copper-binding affinity (37).

Consecutive repetitive application of copper, in the presence of DTT, to a given preparation of isolated mitochondria induced progressive alterations of mitochondrial ultrastructure that were highly similar to those observed in Atp $7 b^{-/-}$mitochondria (compare Figure 5, D-G, and Supplemental Figure 4, C-E, with Figures 2 and 3, Supplemental Figure 2, and Supplemental Figure $3 \mathrm{~A})$. Low copper doses caused rounding and enlargement of the mitochondrial cristae (Figure 5D and Supplementary Figure 4C), while increasing doses caused severe enlargements of the intermembrane spaces (Figure 5, E and F, and Supplemental Figure 4E) and induced matrix remnant accumulation at the mitochondrial membranes (Figure 5G and Supplemental Figure 4F), resulting in structures that resembled those found in the pellet fraction of mitochondria from clinically apparent $A t p 7 b^{-1-}$ rats (Figure 3B).

The conspicuous equivalence between the structural alterations of control mitochondria incubated in vitro with copper plus DTT

\section{Figure 4}

Oxidative damage in $A t p 7 b^{-/-}$mitochondria occurs at a late disease stage. (A) No early mitochondrial oxidative damage occurs at the level of the redox-sensitive mitochondrial aconitase in Atp $7 b^{-/-}$animals in comparison to control animals (the higher activity in the age group $60-61$ days may be due to the younger animal age). Mitochondria from clinically apparent $A t p 7 b^{-/-}$animals showed markedly lower activities. Mitochondria were isolated from controls (86-114 days, 4 measurements) and $A t p 7 b^{-/-}$rats (60-61 days, 3 measurements; 81-89 days, 5 measurements; clinically apparent, 106-113 days, 2 measurements; D-PA-treated, 121-122 days, 4 measurements). (B) Unsaturated mitochondrial fatty acids are depleted only at a late stage of WD progression in $A t p 7 b^{-/-}$rats. The major abundant fatty acids, accounting for $94 \%$ of the typical mitochondrial fatty acid composition (values from control mitochondria given in parentheses) are displayed. Fatty acids were isolated from mitochondria from $A t p 7 b^{-/}$animals at the ages of $60-61$ days $(n=3), 72-73$ days $(n=3)$, and $85-86$ days $(n=3)$ and from clinically apparent $A t p 7 b^{-/-}$animals at age 86-100 days $(n=8)$. Fatty acid amounts are given relative to values obtained from control mitochondria $(n=3)$ set to $100 \%$. (C) Enzymatic activities in mitochondria isolated from controls ( $85-86$ days, $n=6$ ) were highly similar to activities in mitochondria from Atp $7 b^{-/-}$animals (88-89 days, $n=5$ ) and only slightly altered in mitochondria from clinically apparent animals (92-99 days, $n=5$ ). A significant decrease in activities occurs in mitochondria from the pellet fraction of clinically apparent animals (88-103 days, $n=3$ ). ${ }^{\star} P<0.05,{ }^{\star \star} P<0.01,{ }^{* \star *} P<0.001$.
$6 \mathrm{~B})$, and were recovered in the pellet fraction (Figure 6C).

and the mitochondrial phenotypes of Atp $7 b^{-/-}$animals in vivo suggested that this cell-free system was an appropriate model for studying the functional deficits of such altered mitochondria (Figure 6). Mitochondria were able to accumulate rather large doses of copper without manifesting severe functional deficits, provided that they were kept in the presence of reducing thiol equivalents (Figure 6). However, when the molar ratios of copper/DTT came close to equivalence, mitochondria abruptly dissipated their transmembrane potential (Figure 6A), lost their capacity to produce ATP (Figure Identification of copper-reactive mitochondrial thiol proteins indicate intramitochondrial (membrane) protein crosslinking. Since copper reacts with thiol-bearing molecules, such as cysteines (38), GSH (39), and
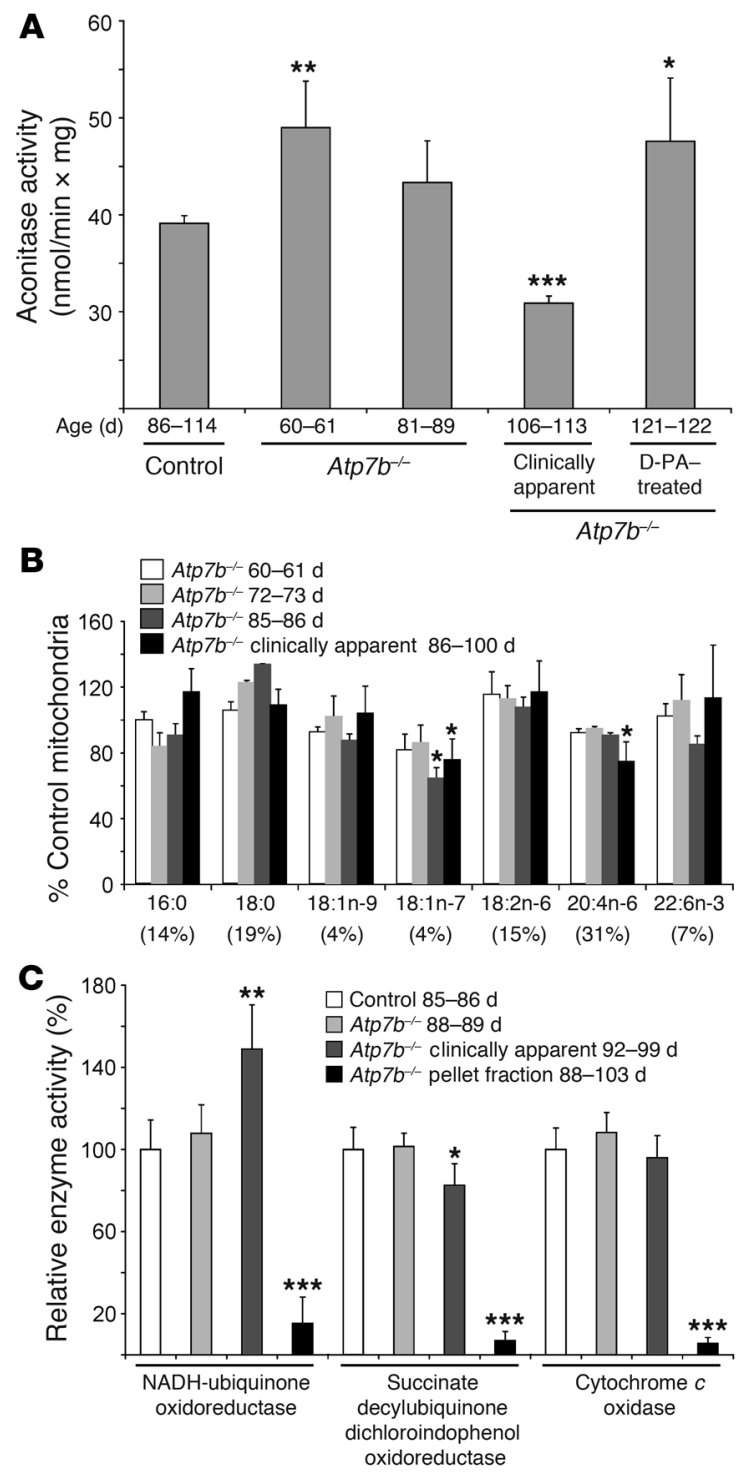

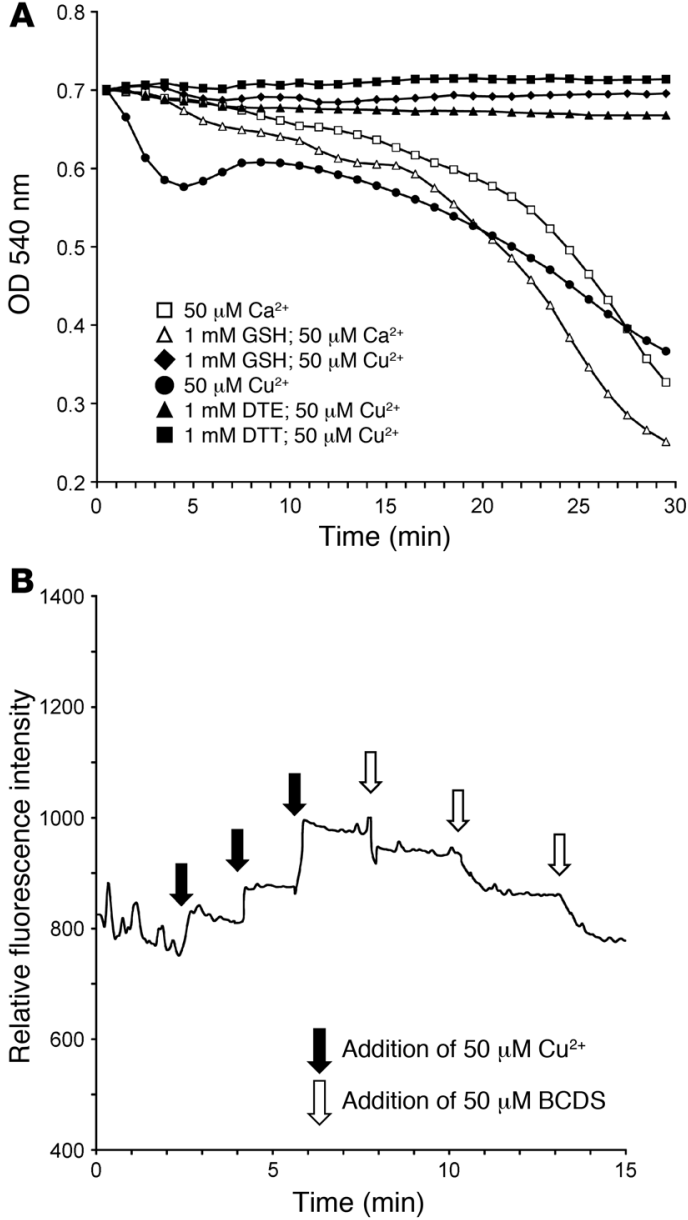
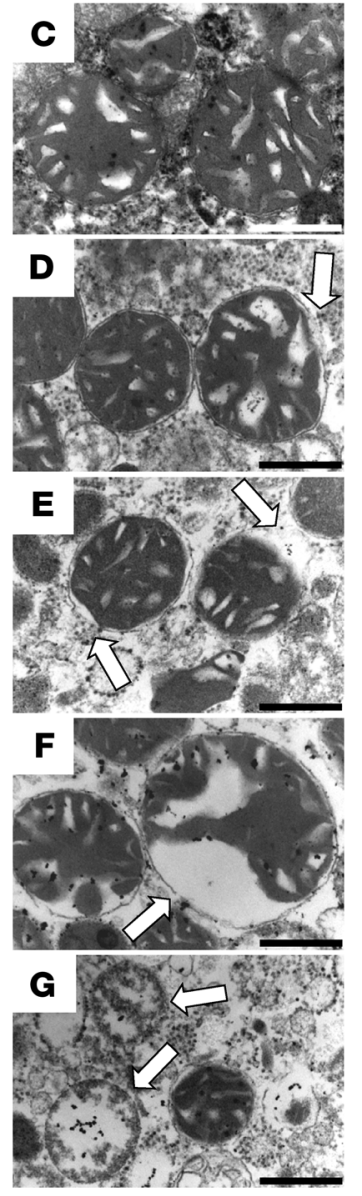

\section{Figure 5}

Simulation of pathological mitochondrial alterations in a cell-free system. (A) Copper and calcium induce mitochondrial swelling as assessed by optical density measurements of mitochondrial suspensions at $540 \mathrm{~nm}$. In contrast to calcium, copper-triggered swelling could be inhibited by reduced GSH and dithiols such as DTT and DTE. Each data curve represents the average of 4 individual measurements for copper and duplicates for calcium. (B) In the presence of DTT, repetitive copper dosing induced a staircase-like increase in light refraction, indicative of mitochondrial contraction. This increase could be reversed by repetitive equimolar doses of the copper chelator BCDS. Measurements of mitochondrial suspensions were performed at $540 \mathrm{~nm}$ in a fluorescence spectrometer. For clarity, fluorescence peaks due to additions made during the measurement were removed from the displayed graph. (C-G) Electron micrographs of control mitochondria treated with copper in the presence of DTT. Note that copper was added repeatedly to the same mitochondrial suspension and samples withdrawn after the additions: (C) untreated starting control, (D) $100 \mu \mathrm{M}$ copper/1 mM DTT, (E) $200 \mu \mathrm{M}$ copper/1 mM DTT, (F) $300 \mu \mathrm{M}$ copper/1 mM DTT, (G) $400 \mu \mathrm{M}$ copper/1 mM DTT. Mitochondria with progressively enlarged cristae and intermembrane spaces were found. At high copper doses matrix remnants gathered at the mitochondrial membranes (arrows). Scale bars: $0.5 \mu \mathrm{m}$.
DTT (40), we hypothesized that the reversible mitochondrial structural alterations observed in vitro (Figure 5B and Supplemental Figure 4B) might be due to rapidly formed mixed copper-DTT-thiol complexes, involving cysteines in mitochondrial proteins. Due to the dithiol character of DTT, copper-DTT complexes might act as "crosslinkers" that connect proteins within the same mitochondrial compartment or the same membrane or on distinct mitochondrial membranes. To identify mitochondrial membrane proteins that react with copper-DTT complexes, we employed a redox proteomics approach (41). Mitochondria were either treated with copper-DTT or left untreated as controls, and their membrane proteins were enriched by carbonate extraction. Free thiols that did not react with copper-DTT were covalently modified with a fluorescent adduct, and the mixed fluorescent and non-fluorescent proteins were separated by BAC/SDS-PAGE (Supplemental Figure 5 and refs. 41, 42). We then used mass spectrometry to identify those proteins whose fluorescence was reduced upon challenge with copper-DTT (indicating that their thiols had been modified by copper-DTT) and found the voltage-dependent anion channel (VDAC), the adeninenucleotide translocator (ANT), and the B subunit of ATP synthase (Figure 6D, arrows) as targets of copper-DTT-induced reactions. It should be noted that the overall abundance of these copper-DTTmodified proteins did not change upon treatment (as revealed by silver staining, Figure 6D).

Mitochondrial membrane crosslinking upon copper accumulation in vivo. To investigate whether intramitochondrial membrane crosslinking occurred in $A t p 7 b^{-/-}$mitochondria, we subjected isolated mitochondria from $A t p 7 b^{-/-}$animals to osmotically driven swelling/shrinking conditions, followed by mild shearing. Such treatments typically strip off parts of the outer membrane from mitochondria, leading to the generation of mitoplasts (43). As expected, control mitochondria lost outer membrane parts under such conditions, as assessed by a marked depletion of VDAC (Figure 7A). In contrast, VDAC depletion was absent in mitoplasts derived from mitochondria from $A t p 7 b^{-/-}$rats (Figure 7A). Rather, these mitoplasts tended to keep large parts of outer membrane relics attached (Figure 7B).

Next, we subjected isolated $A t p 7 b^{-/-}$mitochondria to calcium treatments eliciting MPT. Such treatments cause mitochondrial swelling and outer membrane rupture (Figure 5A). The extent of outer membrane rupture in swollen mitochondria was analyzed by zone electrophoresis in a free-flow electrophoresis (ZE-FFE) device. The more the outer membrane is stripped off by MPT from the mitochondria, the more the resulting remnants shift toward the anode (43). Control mitochondria treated with high-dose calcium presented two major peaks (M3 and M4) deflecting toward the anode in ZE-FFE (Figure 7C). These M3 and M4 mitochondria had lost most of their outer membranes, as indicated by depletion of VDAC and the intermembrane protein cytochrome $c$ (Figure 7D). Atp $7 b^{-/-}$mitochondria incubated with calcium also showed an anodal shift in ZE-FFE, but with one major peak (M1), demonstrating that these mitochondria underwent MPT (Figure 7C). However, M1 mitochondria deflected much less toward the anode 

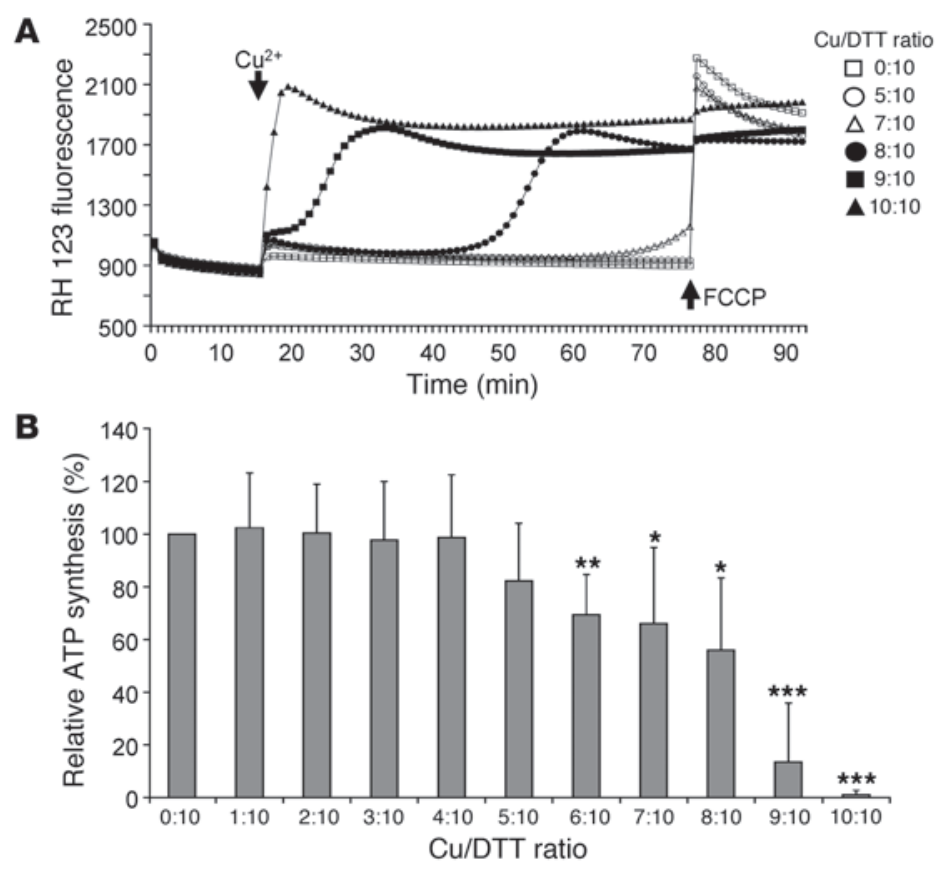

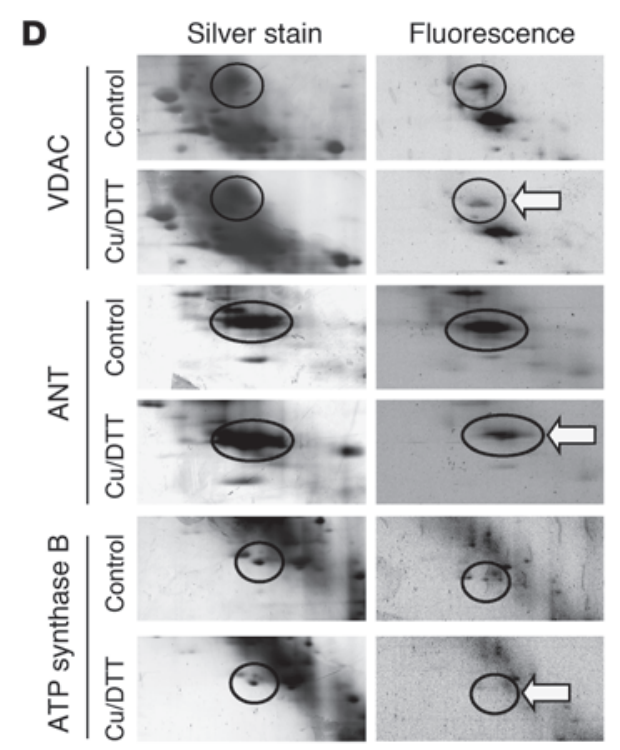

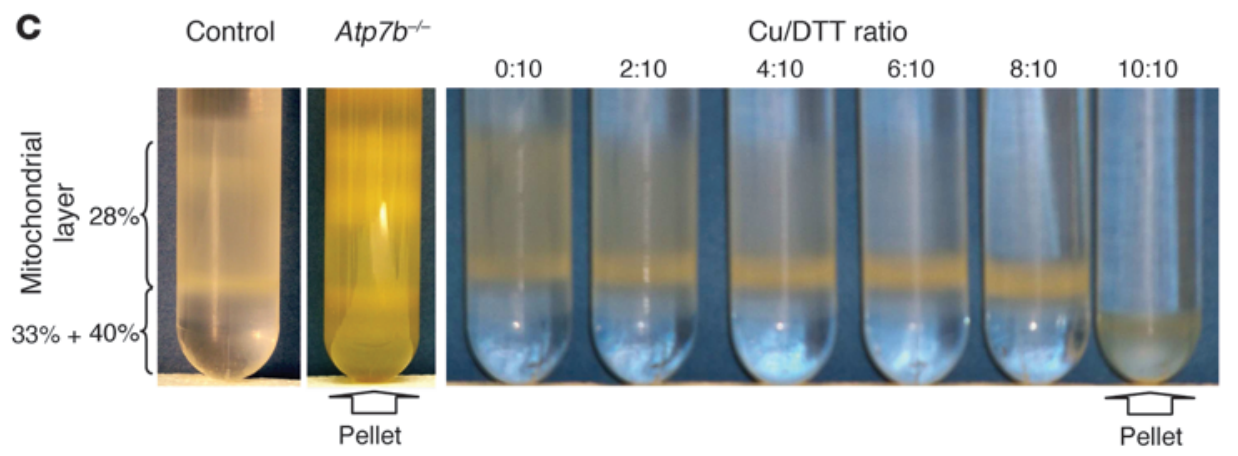

\section{Figure 6}

Increasing copper/DTT ratios impair mitochondrial function and membrane proteins. (A) Increasing copper/DTT ratios compromise $\Delta \psi_{\mathrm{m}}$, as assessed by fluorescence quenching of Rh123, and induce a complete immediate $\Delta \psi_{\mathrm{m}}$ loss at ratios close to parity (Cu/DTT 10:10). DTT alone had no effect on $\Delta \psi_{\mathrm{m}}(\mathrm{Cu} / \mathrm{DTT}$ 0:10). Different amounts of copper were added to reach the indicated increasing copper/DTT ratios. Carbonyl cyanide 4-(trifluoromethoxy)phenylhydrazone (FCCP) served as an internal control for complete $\Delta \psi_{m}$ dissipation. Each data curve represents the average of 4 individual measurements. (B) Mitochondrial ATP synthesis capacity is impaired at an increasing copper/DTT ratio and completely lost at copper/DTT ratios close to parity. Data represent the average of 5 independent experiments. (C) Upon consecutively increasing copper/ DTT ratios, liver mitochondria gather at the border of the higher-density solution. Further copper addition leads to mitochondrial accumulation in this solution and the pellet fraction. This pellet fraction is also observed in clinically apparent $A t p 7 b^{-/-}$animals, but not in control rats. (D) The mitochondrial membrane proteins VDAC, ANT, and ATP synthase B react with copper/DTT. These proteins were found to be altered in thiol status in 3 independent replicates. Mitochondria were either incubated with copper/DTT or left untreated as controls. Mitochondrial membrane proteins were enriched by carbonate extraction, and thiol residues were stained with the fluorophore BODIPY FL. Protein extracts were separated by BAC/SDS-PAGE, scanned for determination of the protein-bound fluorescence, and finally silver stained for protein amount comparisons. Proteins were identified by mass spectrometry. ${ }^{\star} P<0.05,{ }^{\star *} P<0.01,{ }^{\star \star *} P<0.001$.

than M3 or M4 mitochondria (Figure 7C). In agreement with this result, calcium-treated $A t p 7 b^{-/-} \mathrm{M} 1$ mitochondria contained almost as much VDAC as untreated mitochondria, but demonstrated a slight depletion of cytochrome $c$ (Figure 7D).

Together, these results indicate that the outer membranes of mitochondria from $A t p 7 b^{-/-}$rats are attached more tightly to the organelles than those of mitochondria from control animals.

Copper-chelating therapy restores mitochondrial structure and function in Atp $7 b^{-1-}$ rats. To address the question of whether the structural alterations in WD mitochondria are reversible in vivo, as was observed in vitro (Figure 5B and Supplemental Figure 4B), we treated Atp $7 b^{-/-}$ rats with the copper chelator D-PA, the treatment of choice for WD $(5,6,44)$, or with the copper-chelating peptide methanobactin. The treatment was started at day $84 / 85$ of age ( $n=4$ for each treatment). In addition, 2 animals with incipient liver damage (AST > $200 \mathrm{mU} /$ $\mathrm{ml}$ ) were D-PA-treated from day 92 onward.

Seven of the $8 A t p 7 b^{-/-}$rats treated from day $84 / 85$ on remained healthy as indicated by a constant gain in weight, normal plasma levels of AST, and normal liver histology (examples shown in Supplemental Figure 6), whereas untreated $A t p 7 b^{-/-}$animals 
A
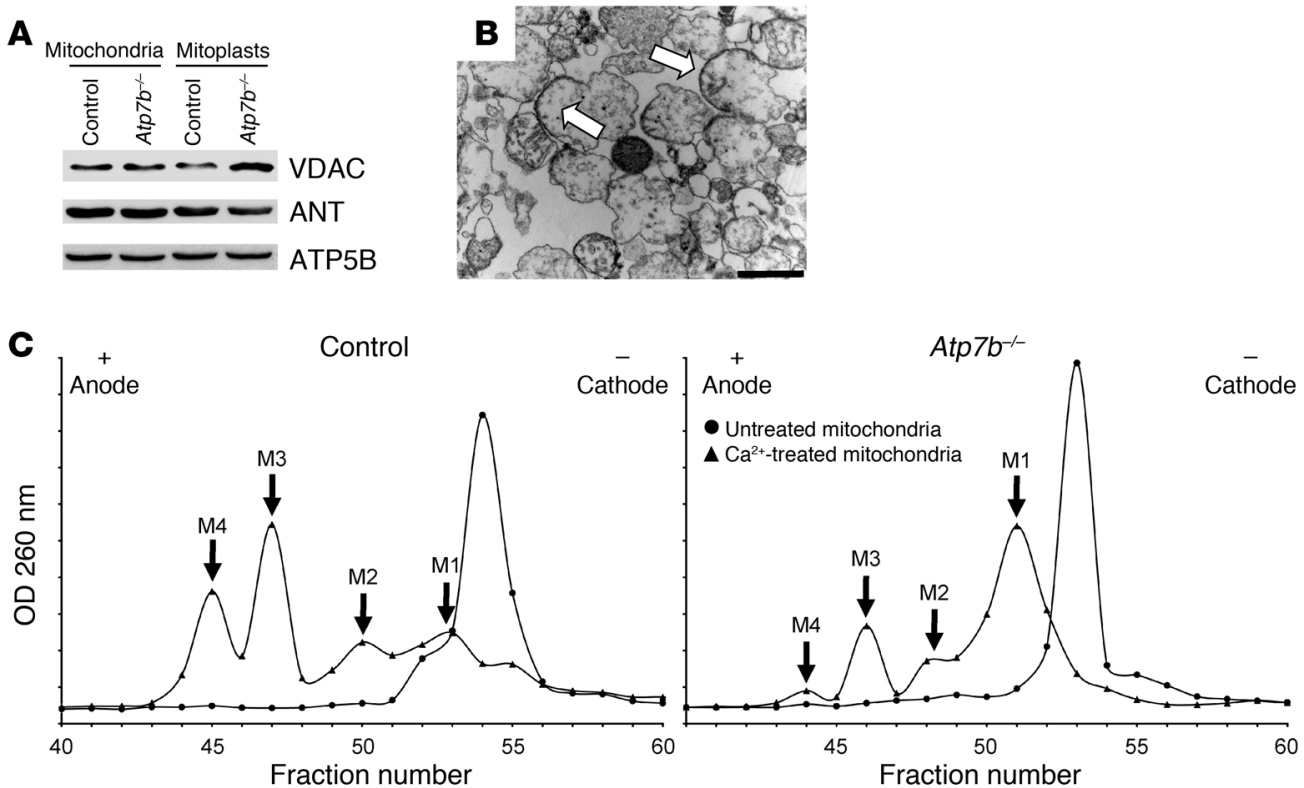

D

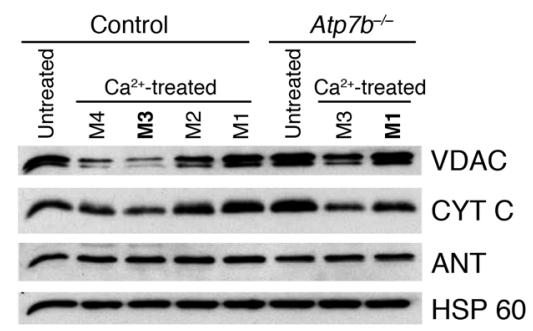

Figure 7

Intramitochondrial membrane crosslinking can be detected in Atp $7 b^{-/-}$mitochondria. (A) Mitochondria from Atp $7 b^{-/-}$animals and controls were subjected to hypo- and hyperosmotic conditions with subsequent shearing, resulting in the formation of mitoplasts. Control mitochondria but not their counterparts from Atp $7 \mathrm{~b}^{-/-}$livers lost parts of their outer membrane, as demonstrated by a marked depletion of the outer membrane VDAC. The inner membrane markers ANT and ATP5B served as loading controls. (B) Electron micrograph of mitoplasts generated from $A t p 7 b^{-1-}$ mitochondria as described in A. Outer membrane relics were attached to these structures (arrows; scale bar: $1 \mu \mathrm{m}$ ). (C) ZE-FFE analysis of mitochondria from control and Atp7 $7 \mathrm{~b}^{-/-}$ animals subjected to $\mathrm{Ca}^{2+}$-induced MPT. Control mitochondria treated with calcium presented two major peaks, M3 and M4, deflecting strongly toward the anode in comparison to untreated mitochondria. In contrast, calcium-treated $A t p 7 b^{-/-}$mitochondria presented a much less pronounced anodal shift in ZE-FFE, with M1 mitochondria as major fraction. (D) Immunoblot analysis of the separated mitochondrial subpopulations of C. M3 and M4 mitochondria had lost large amounts of their outer membrane, as indicated by depletion of VDAC and losses of cytochrome $c$ (CYT C). M1 mitochondria did contain almost equal amounts of VDAC as compared with untreated mitochondria yet demonstrated a partial depletion of cytochrome $c$, indicative of outer membrane rupture. The major fraction of $\mathrm{Ca}^{2+}$-treated control mitochondria was $\mathrm{M} 3$ and that of Atp $7 b^{-/-}$mitochondria was M1 (marked in bold).

manifested the typical signs of hepatic cell death (Figure 1B). One animal in the methanobactin-treated group as well as one animal with incipient liver damage developed signs of severe liver failure (Supplemental Figure 6, E and F), and these had to be sacrificed at day 113 and 106, respectively.

Copper chelation in the positively responding $A t p 7 b^{-/-}$animals treated from day $84 / 85$ onward resulted, as was described for D-PA (45), in a reduction in the copper burden (Figure 8A). Unexpectedly, however, this reduction preferentially occurred in mitochondria, not in the cytosol and slightly in lysosomes (Figure 8A).

The mitochondrial copper depletion was paralleled by a return to normal morphology in treated mitochondria (Figure 8, B and C), liver mitochondria from $A t p 7 b^{-1-}$ animals.

In a first phase of WD, a continually increasing mitochondrial copper load, coinciding with mitochondrial structure alterations (Figure 2 and Supplemental Figure 2) occurred well before WD symptoms became clinically apparent (compare the kinetics in Figure 2, E and F, with Figure 1B). Liver tissues of WD patients $(23,24)$ and $A t p 7 b^{-/-}$rats (Supplemental Figure 2A) exhibited characteristic changes in mitochondrial ultrastructure, with massive enlargements of intermembrane spaces and severe condensations within the organelles. Such vast qualitative and quantitative structural alterations were preserved upon mitochondrial isolation (Figure 2 and Supplemental Figure 2), while control mitochondria presented 
A
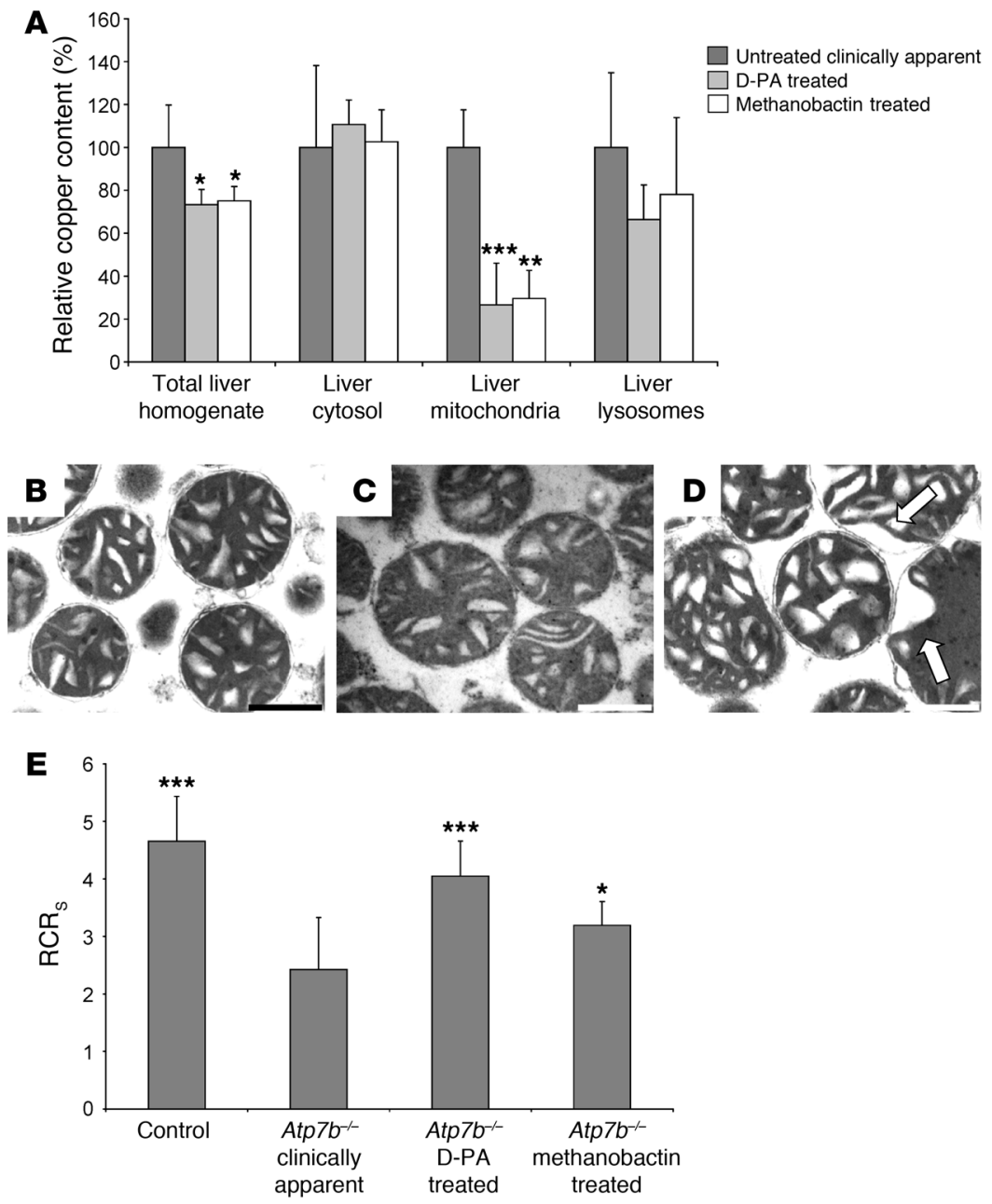

\section{Figure 8}

Copper chelation therapies restore mitochondrial structure and function in $A t p 7 b^{-/-}$rats. (A) The copper chelators D-PA and methanobactin decrease the copper content in liver compartments of $A t p 7 b^{-/}$animals ( $n=4$ and $n=3$, respectively), preferentially in mitochondria. Copper amounts in liver subfractions are expressed relative to values from untreated clinically apparent $A t p 7 b^{-/-}$animals $(n=4)$ set to hundred percent. Electron micrographs of liver mitochondria isolated from (B) a D-PA-treated and (C) a methanobactin-treated healthy $A t p 7 b^{-/-}$animal (122 days and 120 days old, respectively; treatment started at day 85 ) and (D) an Atp7 $b^{-/-}$rat with initial liver damage (129 days old, D-PA treatment started at day 92). Whereas structural integrity was observed in $\mathbf{B}$ and C, slight enlargements of the intermembrane spaces were observed in $\mathbf{D}$, indicating incomplete mitochondrial recovery (arrows). Scale bars: $0.5 \mu \mathrm{m}$. (E) D-PA and methanobactin treatments restore mitochondrial functional activity, as assessed by the succinate-driven respiratory control ratio $\left(\mathrm{RCR}_{\mathrm{S}}\right)$ in $A t p 7 b^{-/-}$animals. A significantly higher $\mathrm{RCR}_{S}$ was detected in mitochondria from $A t p 7 b^{-1-}$ animals that positively responded to these treatments versus clinically apparent $A t p 7 b^{-/-}$ rats. ${ }^{*} P<0.05,{ }^{* *} P<0.01,{ }^{* *} P<0.001$. only minor structural heterogeneities, now and then with some mitochondria showing structural deficits (Figure 2E). Therefore, the intramitochondrial condensation of isolated $A t p 7 b^{-/-}$mitochondria is not a consequence of the isolation process but reflects a molecular event that has occurred in vivo.

During the second phase of WD, Atp $7 b^{-1-}$ hepatocyte damage became clinically apparent (Figure $1 \mathrm{~B}$ ). This coincided with the isolation of severely impaired mitochondria (Figure 3 and Supplemental Figure 3) in a massively copper-laden pellet fraction (Figure $2 \mathrm{~F}$ ), possibly explaining their abnormally high buoyant density (Figure 6C). This pellet fraction contained mitochondria with greatly enlarged intermembrane spaces (Figure 3A), severely transformed mitochondria with electron-dense matrix remnants gathering at the membrane (Figure 3, B and C, and Supplemental Figure $3 \mathrm{~A}$ ), and smaller vesicles resembling these structures (Figure $3 \mathrm{~B}$ ), indicating their mitochondrial origin. This conclusion was supported by the reproduction in vitro of both intermembrane space enlargements and matrix condensations at the membranes in isolated control mitochondria upon addition of copper (Figure 5, F and G). Moreover, the copper load measured in the pellet fraction isolated from $A t p 7 b^{-1-}$ rats $(6.7 \pm 2.8 \mu \mathrm{g}$ copper per mg mitochondrial protein; Figure $2 \mathrm{~F}$ ) matched the dose of copper $(4.7-6.3 \mu \mathrm{g} / \mathrm{mg})$ that induced these alterations in vitro. Thus, these mitochondrial fragments/remnants are induced upon mas- sive copper load both in vitro and in vivo. Since their occurrence coincided with the abrupt onset of liver failure in $A t p 7 b^{-/-}$animals, it appears that the second, clinically apparent WD phase is marked by severe mitochondrial impairments and destruction.

Addition of copper to isolated mitochondria induced immediate mitochondrial contraction, provided that the organelles were kept in the presence of DTT (Figure 5B and Supplemental Figure 4B). Such thiol-reducing agents mimic the physiological intramitochondrial milieu, which contains millimolar GSH (46). Moreover, mitochondria normally contain approximately 65-70 nmol thiol residues/mg mitochondrial protein, one-third of which are exposed and reactive (47). Thus, in vivo, reduced thiols are available in large amounts in mitochondria. Interestingly, the addition of the copper chelators BCDS and methanobactin reversed the mitochondrial contraction in the cell-free system (Figure 5B and Supplemental Figure 4B), underscoring that the prime cause for mitochondrial condensation is copper. Accordingly, treatment of Atp $7 b^{-/-}$animals with copper chelators lead to preferential depletion of copper from mitochondria and restored mitochondrial ultrastructure (Figure 8). This demonstrates that mitochondria are a primary target of copper in WD. The observations that the copper-induced mitochondrial condensations were reversible in vivo and in vitro, and that they were favored by reducing conditions in the cell-free system, clearly argue against the possibility that cop- 
per would trigger irreversible oxidative stress by Fenton chemistry to alter mitochondrial morphology and function in early WD. Indeed, we failed to observe significant signs of oxidative damage in $A t p 7 b^{-1-}$ mitochondria before WD became clinically apparent (Figure 4, A and B; see below). Thus, the copper-driven intramitochondrial condensations that characterize the early phase of WD are not associated with substantial oxidative stress.

Could the copper-induced, reversible mitochondrial contraction observed in vitro be explained by rapidly formed mixed copperDTT-thiol complexes involving cysteines of mitochondrial proteins? In a redox proteomics approach focusing on mitochondrial membrane proteins, we indeed identified thiol modifications in 3 abundant mitochondrial membrane proteins: VDAC, ANT, and ATP synthase B (Figure 6D). It is tempting to speculate that the mitochondrial structural alterations elicited by copper-DTT may result from intermolecular thiol bridges between proteins anchored in the outer membrane (such as VDAC) and the inner membrane (such as ANT). Indeed, multiple mitochondrial proteins, including ANT, are known to contain reactive dithiols (48). In this hypothetical scenario, as the mitochondrial inner membrane contracts, the cristae and intermembrane space enlarge. Within discrete areas of mitochondria, the contracted inner membrane, and possibly the matrix, condenses in close proximity to the outer membrane, thus offering a biochemical explanation for the observed ultrastructural changes. Since VDAC, ANT, and ATP synthase are among the most abundant mitochondrial proteins, they may represent the tip of the iceberg, and additional large-scale analyses may lead to the identification of additional copper-reactive proteins within mitochondria. In fact, we obtained additional evidence supporting the idea that copper accumulation in mitochondria causes increased interaction between the outer and inner mitochondrial membranes. Calcium-induced MPT as well as osmotic and shear stress removed less outer membrane from $A t p 7 b^{-/-}$mitochondria than from control organelles (Figure 7). Moreover, attempts to isolate pure mitochondrial outer membranes from clinically apparent $A t p 7 b^{-1-}$ rats reproducibly failed, although in the same conditions outer membranes could be purified from control mitochondria (H. Zischka, unpublished observations). These findings strongly support the notion of copper-enforced intramitochondrial membrane interactions in $A t p 7 b^{-/-}$rats. It appears plausible that the structural alterations occurring instantly in vitro also occur in vivo - though possibly with slower kinetics - upon interaction of copper with cysteine-bearing proteins.

Loss of mitochondrial function, which characterizes the second phase of WD (Figure 4C) could be reproduced in vitro at particularly high copper/DTT ratios (Figures 6, A-C). Copper reacts with DTT in two steps (40). Initially, copper-DTT complexes rapidly form in a reversible and ROS-independent fashion. Then, the copper-DTT complexes oxidize and generate ROS $(38,40)$. This ROS production, however, is characterized by comparatively slow kinetics and can be significantly inhibited by a molar excess of DTT over copper (40), as reflected by low copper/DTT ratios in our cell-free system. When the copper amounts increase and DTT gets exhausted, massive oxidative stress results (i.e., high copper/DTT ratios in our cell-free system). Thus, the observed mitochondrial impairments and destruction in vitro may be attributed to high copper-induced oxidative stress. In fact, massive signs of oxidative stress were detected in $A t p 7 b^{-/-}$mitochondria isolated from clinically apparent animals (Figure 4, A and B). We thus conclude that exhaustion of the mitochondrial copper-binding sites together with massive copper-protein aggregates at the mitochondrial membranes could sensitize $A t p 7 b^{-1-}$ mitochondria toward sudden copper-induced destruction, leading to hepatocyte cell death and possibly explaining acute hepatic failure in WD. Indeed, in preliminary studies, we observed that isolated mitochondria fragmented upon exposure to extensive oxidative stress conditions imposed by a combined treatment with copper and ascorbate (H. Zischka, unpublished observations). Future studies must determine whether this fragmentation is directly mediated by leaking free copper and to what extent it marks an irreversible event in the pathogenic cascade of WD.

In summary, the major conclusion of this study is that liver failure in WD is due to structural and functional mitochondrial impairment induced by excessive mitochondrial copper load. Mitochondria are the early and immediate target for a changing cellular copper burden. In the initial phase of the disease, the increase in mitochondrial copper content is paralleled by major structural alterations but only minor functional deficits. Since these early alterations could be reversed by copper-chelating therapies in vivo and in vitro, it seems doubtful that this mitochondrial damage is catalyzed by copper-based Fenton chemistry. Our data point to an alternative explanation: namely, a direct attachment of copper to mitochondrial protein cysteines (and possibly other residues) leading to multivalent interactions or multiprotein crosslinking. In the late phase of the disease, the mitochondrial copper load exceeds normal levels more than 100 -fold, paralleled by massive functional impairments, clear signs of oxidative damage, and mitochondrial destruction, thus leading to clinically apparent liver damage.

\section{Methods}

Animals. LEC rats were from Charles River, and the LPP rat strain was provided by Jimo Borjigin, University of Michigan, Ann Arbor, Michigan, USA. These animals are a crossbreed between LEC rats showing a deletion in the Atp7b gene and PVG rats (33). Rats were maintained on an ad lib Altromin 1314 diet (Altromin Spezialfutter $\mathrm{GmbH}$ ) and tap water. The copper content of the rat diet was $13 \mathrm{mg} / \mathrm{kg}$. All animals were treated under the guidelines for the care and use of laboratory animals of the Helmholtz Center Munich.

Animal treatments. Atp $7 b^{-/-}$rats were treated for 5 weeks with D-PA or methanobactin at a dose of $100 \mathrm{mg} / \mathrm{kg} \mathrm{BW} / \mathrm{d}$ in the drinking water or by i.p. injections at a dose of $62.5 \mathrm{mg} / \mathrm{kg} \mathrm{BW} 3$ times per week (16 treatments in total), respectively $(45,49)$. Animal experiments were verified by the Ausschuss für Tierversuche und Versuchstierhaltung (ATV) at Helmholtz Center Munich and approved by the ethical committee of the Regierung von Oberbayern, Munich.

Histopathologic examination. Formalin-fixed, paraffin-embedded liver samples were cut into 4- $\mu \mathrm{m}$-thick sections and stained with hematoxylin and eosin.

Plasma AST and bilirubin. AST activity and bilirubin concentration in animal plasma were measured with a Reflotron system (Roche).

Liver subfractionation and isolation of mitochondria. Mitochondria, lysosomes, and cytosol were derived from freshly prepared liver homogenates as described previously (34). Specifically, mitochondria were purified by differential centrifugation, with $2-3$ additional washing steps implemented for experiments in Figure 5, Figure 6D, Supplemental Figure 4A, Supplemental Figure 4, C-F, and Supplemental Figure 5; by density gradient centrifugation for experiments in Figure 2F, Figure 3B, Figure 4, Figure 6, A-C, Figure 7, A-C, Figure 8, and Supplemental Figure 3; by a combination of density gradient centrifugation and ZE-FFE purification for experiments in Figure 2, A-E, Figure 3A, Figure 3C, Figure 7D, and Supplemental Figure 2, B-E. Fresh mitochondria were used for respiratory measurements or copper and calcium challenge experiments and were sub- 
sequently analyzed for swelling, transmembrane potential $\left(\Delta \psi_{\mathrm{m}}\right)$, and ATP synthesis or subjected to ZE-FFE analysis, electron microscopy, and redox proteomics. Mitochondrial samples isolated by density gradient purification were stored at $-192^{\circ} \mathrm{C}$ in preservation buffer (50) and used for the assessments of enzymatic activities, lipid peroxidation, and quantification of structural alterations. For the latter analysis, samples were subsequently purified by ZE-FFE on the same day and subjected to electron microscopy analysis in parallel.

Metal content determination. Copper and zinc in liver homogenates and mitochondrial preparations were analyzed by ICP-OES (Ciros Vision, SPECTRO Analytical Instruments $\mathrm{GmbH}$ ) after wet washing of samples with $65 \%$ nitric acid.

Mitochondrial analyses. Functional integrity of isolated mitochondria was assessed by standard respiratory measurements (50) in a Clark-type oxygen electrode (Oxygraph, Hansatech Instruments). Mitochondrial enzyme activities were assessed as described previously (51). Kit-based assays were used to analyze aconitase activity (Cayman Chemical) and ATP synthesis (ATP Bioluminescence Assay Kit CLS II, Roche). Mitochondrial swelling was measured by light scattering, either with an absorbance reader in 96-well plate formats or with a cuvette in a fluorescence reader at $540 \mathrm{~nm}$ (43). Assessment of $\Delta \psi_{\mathrm{m}}$ was followed by Rh123 fluorescence quenching according to ref. 52 in a 96-well plate reader (BioTek). ZE-FFE analyses of $\mathrm{Ca}^{2+}$-treated mitochondria were done as recently described (43).

Mitochondrial lipid peroxidation. Mitochondrial lipids were extracted with chloroform/methanol (2:1), washed, and evaporated to dryness. Lipids were dissolved in dichloromethane and the fatty acids converted to their methyl esters by Methyl-Prep II (Alltech Associates Inc.). Fatty acid methyl esters extracted and dissolved in hexane were analyzed by capillary gas chromatography (Agilent Technologies). Identification of fatty acid methyl esters was obtained by comparison with the retention time of pure standard mixtures (Sigma-Aldrich). The relative amount of each fatty acid methyl ester was expressed as the percentage of total area.

Mitochondrial redox proteomics. Freshly isolated mitochondria were treated with DTT $(1 \mathrm{mM})$ and $\mathrm{CuSO}_{4}(200 \mu \mathrm{M})$ for 15 minutes. Mitochondrial membrane proteins were enriched from these samples by carbonate extraction (42) and solubilized with $1 \%$ DDM. Proteins were labeled with $15 \mathrm{nmol}$ BODIPY FL/50 $\mu \mathrm{g}$ protein (Invitrogen). Dye surplus was removed with Micro Bio-Spin 6 columns (Bio-Rad). 16-BAC/PAGE was carried out essentially as described previously (42) in a PROTEAN II Gel System (Bio-Rad), but without thiol-reducing agents. Protein $(50-100 \mu \mathrm{g}$ per lane) was loaded on $12 \%$ BAC gels, equilibrated without reducing or alkylating reagents, and run in second-dimension $8 \%-16 \%$ SDS-PAGE. Separated proteins were visualized by silver staining and their free thiol residues by fluorescence scanning using the FLA-3000 phosphorimaging system (Fuji Photo Film
$\mathrm{GmbH}$ ) with the BASReader version 3.01 (Raytest) with excitation wavelength of $473 \mathrm{~nm}$. Proteins of interest were analyzed by mass spectrometry of retrieved tryptic peptides essentially as described previously $(53,54)$ on a MALDI-TOF/TOF tandem mass spectrometer (ABI 4700 Proteomics Analyzer, Applied Biosystems). Obtained spectra were processed and proteins identified using the ABI GPS Explorer to search the Swiss-Prot database (http://expasy.org/sprot/).

Miscellaneous. Electron microscopy of liver tissues and mitochondria was done as previously described (43). Methanobactin was isolated from the spent media of Methylosinus trichosporium OB3b as previously described (55). Quantification of mitochondrial protein was determined by the Bradford assay. Immunoblotting analysis of protein extracts was carried out according to Towbin et al. (56). Equal protein amounts were applied per lane, and blots were stained with Ponceau red to control for proper transfer. For protein detection antisera against VDAC (Acris Antibodies), cytochrome $c$ and HSP60 (BD), COXIV (Cell Signaling Technology), CYPD (MitoSciences Inc.), ANT (Santa Cruz Biotechnology Inc.), and ATP5B (Aviva Systems Biology) were used.

Statistics. Data are presented as mean and SD. For Student's $t$ test, data were tested unpaired and 2-tailed, except for those represented in Figure $2 \mathrm{~F}$ and Figure 8A (unpaired and 1-tailed) and Figure 6B (paired and 1-tailed). Differences were considered statistically significant when $P$ values were less than 0.05 .

\section{Acknowledgments}

The support of E. Rojo, P. Reitmeir, M. Aichler, T. Klein-Rodewald, C. Schempp, and the Core Facility Proteomics at HMGU is gratefully acknowledged. G. Kroemer is supported by the Ligue Nationale contre le Cancer (Equipe labellisée), Agence Nationale pour la Recherche (ANR), the European Commission (APO-SYS, ChemoRes, ApopTrain, Active p53), Fondation pour la Recherche Médicale (FRM), Institut National du Cancer (INCa), Cancéropôle Ile-de-France, and an AXA Chair on Longevity Research. A.A. DiSpirito is supported by the National Science Foundation, USA, grant CHE-10112271.

Received for publication October 12, 2010, and accepted in revised form January 5, 2011.

Address correspondence to: Hans Zischka, Institute of Toxicology, Helmholtz Center Munich, German Research Center for Environmental Health, Ingolstaedter Landstrasse 1, 85764 Munich-Neuherberg, Germany. Phone: 49.89.3187.2663; Fax: 49.89.3187.3449; E-mail: zischka@helmholtz-muenchen.de.
1. Sternlieb I. Wilson's disease. Clin Liver Dis. 2000; 4(1):229-239.

2. Bull PC, Thomas GR, Rommens JM, Forbes JR, Cox DW. The Wilson disease gene is a putative copper transporting P-type ATPase similar to the Menkes gene. Nat Genet. 1993;5(4):327-337.

3. Tanzi RE, et al. The Wilson disease gene is a copper transporting ATPase with homology to the Menkes disease gene. Nat Genet. 1993;5(4):344-350.

4. Gitlin JD. Wilson disease. Gastroenterology. 2003; 125(6):1868-1877.

5. Walshe JM. Penicillamine, a new oral therapy for Wilson's disease. Am J Med. 1956;21(4):487-495.

6. Walshe JM. Wilson's disease; new oral therapy. Lancet. 1956;270(6906):25-26.

7. Wilson SAK. Progressive lenticular degeneration: a familial nervous disease associated with cirrhosis of the liver. Lancet. 1912;179(4626):1115-1119.

8. Cumings JN. The copper and iron content of brain and liver in the normal and in hepato-lenticular degeneration. Brain. 1948;71(pt. 4):410-415.

9. Sokol RJ, Devereaux MW, O’Brien K, Khandwala RA, Loehr JP. Abnormal hepatic mitochondrial respiration and cytochrome $\mathrm{C}$ oxidase activity in rats with long-term copper overload. Gastroenterology. 1993;105(1):178-187.

10. Gu M, et al. Oxidative-phosphorylation defects in liver of patients with Wilson's disease. Lancet. 2000; 356(9228):469-474.

11. Britton RS. Metal-induced hepatotoxicity. Semin Liver Dis. 1996;16(1):3-12.

12. Luza SC, Speisky HC. Liver copper storage and transport during development: implications for cytotoxicity. Am J Clin Nutr. 1996;63(5):812S-820S.

13. Rossi L, Lombardo MF, Ciriolo MR, Rotilio G. Mitochondrial dysfunction in neurodegenerative diseases associated with copper imbalance. Neurochem Res. 2004;29(3):493-504.

14. Mansouri A, et al. Premature oxidative aging of hepatic mitochondrial DNA in Wilson's disease.
Gastroenterology. 1997;113(2):599-605.

15 . Sokol RJ, et al. Oxidant injury to hepatic mitochondria in patients with Wilson's disease and Bedlington terriers with copper toxicosis. Gastroenterology. 1994;107(6):1788-1798.

16. Rae TD, Schmidt PJ, Pufahl RA, Culotta VC, O'Halloran TV. Undetectable intracellular free copper: the requirement of a copper chaperone for superoxide dismutase. Science. 1999;284(5415):805-808.

17. Lippard SJ. Free copper ions in the cell? Science. 1999;284(5415):748-749.

18. Cobine PA, Ojeda LD, Rigby KM, Winge DR. Yeast contain a non-proteinaceous pool of copper in the mitochondrial matrix. J Biol Chem. 2004; 279(14):14447-14455.

19. Cobine PA, Pierrel F, Bestwick ML, Winge DR. Mitochondrial matrix copper complex used in metallation of cytochrome oxidase and superoxide dismutase. J Biol Chem. 2006;281(48):36552-36559.

20. Galluzzi L, Blomgren K, Kroemer G. Mitochondrial 
membrane permeabilization in neuronal injury. Nat Rev Neurosci. 2009;10(7):481-494.

21. Kroemer G, Galluzzi L, Brenner C. Mitochondrial membrane permeabilization in cell death. Physiol Rev. 2007;87(1):99-163.

22. Green DR, Kroemer G. The pathophysiology of mitochondrial cell death. Science. 2004; 305(5684):626-629.

23. Sternlieb I. Mitochondrial and fatty changes in hepatocytes of patients with Wilson's disease. Gastroenterology. 1968;55(3):354-367.

24. Sternlieb I. Fraternal concordance of types of abnormal hepatocellular mitochondria in Wilson's disease. Hepatology. 1992;16(3):728-732.

25. Sternlieb I. Electron microscopy of mitochondria and peroxisomes of human hepatocytes. Prog Liver Dis. 1979;6:81-104.

26. Wu J, Forbes JR, Chen HS, Cox DW. The LEC rat has a deletion in the copper transporting ATPase gene homologous to the Wilson disease gene. Nat Genet. 1994;7(4):541-545.

27. Fuentealba IC, Aburto EM. Animal models of copperassociated liver disease. Comp Hepatol. 2003;2(1):5.

28. Sternlieb I, Quintana N, Volenberg I, Schilsky ML. An array of mitochondrial alterations in the hepatocytes of Long-Evans Cinnamon rats. Hepatology. 1995;22(6):1782-1787.

29. Sokol RJ, Devereaux M, Mierau GW, Hambidge KM, Shikes RH. Oxidant injury to hepatic mitochondrial lipids in rats with dietary copper overload. Modification by vitamin E deficiency. Gastroenterology. 1990;99(4):1061-1071.

30. Roberts EA, Robinson BH, Yang S. Mitochondrial structure and function in the untreated Jackson toxic milk (tx-j) mouse, a model for Wilson disease. Mol Genet Metab. 2008;93(1):54-65.

31. Orrenius S, Gogvadze V, Zhivotovsky B. Mitochondrial oxidative stress: implications for cell death. Annu Rev Pharmacol Toxicol. 2007;47:143-183.

32. Ott M, Gogvadze V, Orrenius S, Zhivotovsky B. Mitochondria, oxidative stress and cell death. Apoptosis. 2007;12(5):913-922.
33. Ahmed S, Deng J, Borjigin J. A new strain of rat for functional analysis of PINA. Brain Res Mol Brain Res. 2005;137(1-2):63-69.

34. Zischka $\mathrm{H}$, et al. Isolation of highly pure rat liver mitochondria with the aid of zone-electrophoresis in a free flow device (ZE-FFE). Methods Mol Biol. 2008;424:333-348.

35. Nair J, et al. Apoptosis and age-dependant induction of nuclear and mitochondrial etheno-DNA adducts in Long-Evans Cinnamon (LEC) rats: enhanced DNA damage by dietary curcumin upon copper accumulation. Carcinogenesis. 2005;26(7):1307-1315.

36. Cobine PA, Pierrel F, Winge DR. Copper trafficking to the mitochondrion and assembly of copper metalloenzymes. Biochim Biophys Acta. 2006;1763(7):759-772.

37. Behling LA, et al. NMR, mass spectrometry and chemical evidence reveal a different chemical structure for methanobactin that contains oxazolone rings. J Am Chem Soc. 2008;130(38):12604-12605.

38. Kachur AV, Koch CJ, Biaglow JE. Mechanism of copper-catalyzed autoxidation of cysteine. Free Radic Res. 1999;31(1):23-34.

39. Kachur AV, Koch CJ, Biaglow JE. Mechanism of copper-catalyzed oxidation of glutathione. Free Radic Res. 1998;28(3):259-269.

40. Kachur AV, Held KD, Koch CJ, Biaglow JE. Mechanism of production of hydroxyl radicals in the copper-catalyzed oxidation of dithiothreitol. Radiat Res. 1997;147(4):409-415.

41. Hochgrafe F, Mostertz J, Albrecht D, Hecker M. Fluorescence thiol modification assay: oxidatively modified proteins in Bacillus subtilis. Mol Microbiol. 2005;58(2):409-425.

42. Braun RJ, Kinkl N, Zischka H, Ueffing M. 16-BAC/ SDS-PAGE analysis of membrane proteins of yeast mitochondria purified by free flow electrophoresis. Methods Mol Biol. 2009;528:83-107.

43. Zischka H, et al. Electrophoretic analysis of the mitochondrial outer membrane rupture induced by permeability transition. Anal Chem. 2008;80(13):5051-5058.

44. Schilsky ML. Diagnosis and treatment of Wilson's disease. Pediatr Transplant. 2002;6(1):15-19.
45. Togashi Y, et al. D-penicillamine prevents the development of hepatitis in Long-Evans Cinnamon rats with abnormal copper metabolism. Hepatology. 1992;15(1):82-87.

46. Valko M, Rhodes CJ, Moncol J, Izakovic M, Mazur $M$. Free radicals, metals and antioxidants in oxidative stress-induced cancer. Chem Biol Interact. 2006; 160(1):1-40.

47. Hurd TR, et al. Glutathionylation of mitochondrial proteins. Antioxid Redox Signal. 2005;7(7-8):999-1010.

48. Halestrap AP, Brenner C. The adenine nucleotide translocase: a central component of the mitochondrial permeability transition pore and key player in cell death. Curr Med Chem. 2003;10(16):1507-1525.

49. Kim HJ, et al. Methanobactin, a copper-acquisition compound from methane-oxidizing bacteria. Science. 2004;305(5690):1612-1615.

50. Fleischer S. Long-term storage of mitochondria to preserve energy-linked functions. Methods Enzymol. 1979;55:28-32.

51. Kiebish MA, et al. Lipidomic analysis and electron transport chain activities in C57BL/6J mouse brain mitochondria. J Neurochem. 2008;106(1):299-312.

52. Zamzami N, Metivier D, Kroemer G. Quantitation of mitochondrial transmembrane potential in cells and in isolated mitochondria. Methods Enzymol.2000; 322:208-213.

53. Shevchenko A, Wilm M, Vorm O, Mann M. Mass spectrometric sequencing of proteins silver-stained polyacrylamide gels. Anal Chem. 1996;68(5):850-858.

54. Gharahdaghi F, Weinberg CR, Meagher DA, Imai BS, Mische SM. Mass spectrometric identification of proteins from silver-stained polyacrylamide gel: a method for the removal of silver ions to enhance sensitivity. Electrophoresis. 1999;20(3):601-605.

55. Bandow NL, et al. Isolation of methanobactin from the spent media of methane oxidizing bacteria. Meth Enzymol. In press.

56. Towbin H, Staehelin T, Gordon J. Electrophoretic transfer of proteins from polyacrylamide gels to nitrocellulose sheets: procedure and some applications. Proc Natl Acad SciU S A. 1979;76(9):4350-4354. 